\title{
Optimising water treatment operations with prediction modelling and smart technologies
}

\author{
$\underline{\text { Edoardo Bertone }}^{\mathrm{a}}$, Xuguang Ren ${ }^{\mathrm{b}}$, Oz Sahin ${ }^{\mathrm{a}, \mathrm{c}}$, Rodney Stewart $^{\mathrm{a}}$, Kelvin O'Halloran ${ }^{\mathrm{d}}$ \\ ${ }^{a}$ School of Engineering and Cities Research Institute, Griffith University, Queensland, Australia \\ ${ }^{b}$ School of Information and Communication Technology, Griffith University, Queensland, Australia \\ ${ }^{c}$ Griffith Climate Change Response Program, Griffith University, Queensland, Australia \\ ${ }^{d}$ Seqwater, Queensland, Australia \\ Email: e.bertone@griffith.edu.au
}

\begin{abstract}
Optimising the raw water intake selection is a critical task for multiple source water treatment plants, as this depends on, among others, water quality, pumping costs, and safety considerations. In case of the Mudgeeraba treatment plant in South-East Queensland (Australia), the raw water can be withdrawn by gravity from the small Little Nerang dam, or pumped from the larger Hinze dam. Intake towers with different gates also allow for the plant operators to withdraw the raw water from different depths. Often though, decision on the optimal intake location is taken based on operators' experience, without a real scientific method relying on in-depth considerations on e.g. current water quality at multiple locations and electricity costs. Nevertheless, the large amount of data collected daily at the treatment plant, and at higher frequency in the two reservoirs by remote vertical profilers, provides an opportunity to link water quality with treatment costs and provide numerical evidence for the operators to take a more informed decision.
\end{abstract}

As a consequence, a number of data-driven, chemical and mathematical models linking raw water quality with required chemicals dosages, as well as a model estimating the pumping costs, were developed. As a consequence, it was possible to predict the overall daily variable treatment costs based on raw water quality. Given that the water quality is monitored in real time in the two reservoirs for the whole water column, it was also possible to provide advice on a daily basis on which withdrawal depth and reservoir would lead to the lowest treatment cost.

By running the model with historical data, it was found that for almost any given day, withdrawing water from Little Nerang dam would have been a cheaper option due to the associated energy costs (i.e. no pumping required) being much lower than potential extra treatment costs due to poorer raw water quality; however, traditionally operators prefer to keep it as a backup reservoir in cases of, for instance, power outages and inability to use the Hinze dam pumps. Given that Little Nerang dam, despite smaller, would be able to provide enough water for few months, an increased use of this water source was still recommended, especially around wet seasons. Based on this, a medium-term storage volume prediction model was developed, able to forecast the risk of depletion and spill for Little Nerang dam for the upcoming six weeks based on, among others, proposed daily withdrawal rates and weather forecasts. In this way, plant operators would be able to plan the future withdrawals in a way to reduce the costs, but also to avoid excessive spill or depletion risks; thus effectively achieving a better planning and management of water treatment operations.

Despite the predicted benefits, it is critical to engage with the relevant stakeholders and potential end-users in order to incentivise the deployment of the end products of research projects; often, lack of proper communication and engagement can lead to the majority of the research outcomes to be largely wasted. This can be a major challenge with water planning and management research. For this research project, the engagement process started during the model development stage, by organising meetings and presentations to show the status of the model and ask for feedback and suggestions to improve it. In addition, a graphical user interface was developed, whose graphical contents (e.g. output charts) were decided based on operators' and managers' suggestions.

Finally, given that such interface was still underutilised because it was installed only on a specific computer at the treatment plant (while several operators usually spend most of their working hours conducting field work), a smartphone application with similar contents to the computer interface was also developed. This can finally lead to a regular deployment of the optimisation models, therefore transforming the research outputs in real quantifiable benefits for the water utility.

Keywords: Decision support system, smartphone application, water treatment optimisation 


\section{INTRODUCTION}

Ensuring that high-quality, safe drinking water is delivered to the consumers is pivotal for any bulk water supplier; drinking water treatment plants have the task to achieve such objective by withdrawing raw water from a number of potential sources (e.g. lakes, reservoirs, rivers), processing it, and delivering the treated water to the distribution system. Several treatment stages are typically present in any treatment process (Kawamura, 2000), and maximising the overall treatment efficiency is an intricate task. One of the main challenges is to dose the correct amount of chemicals, based on raw water quality, in order to achieve treated water quality that complies with the standards fixed by the regulators. This is usually done through jar tests based on the expected raw water quality characteristics.

Some treatment plants, such as the Mudgeeraba water treatment plant (WTP), has the capacity to withdraw raw water from multiple sources. This provides more resilience and the potential of selecting the location with the best water quality in order to facilitate the treatment process. It is also possible to develop models which can provide an estimate of the required doses for certain chemicals based on water quality, and related costs (Abdullahi, 2013), to help operators take a decision on the optimal withdrawal location. When developing such models though, it is critical to engage with stakeholders, as operators might not be interested in understanding a complicated model that would not lead to direct benefits for them, and also because there might be other factors other than costs, which would push the decision-makers not to totally rely on the model's outputs. For the Mudgeeraba case-study, for instance, stakeholder engagement allowed to understand that operators would rarely use one of the two reservoirs despite potentially better water quality, due to some perceived risks (e.g. potential depletion) which scientifically and mathematically seemed unlikely or at least overestimated.

As a result, the overall objectives of this research were:

1. To collect and analyse data for the Mudgeeraba WTP and the two connected reservoirs, namely Hinze dam and Little Nerang dam

2. To develop a model able to quantify the required chemicals dosage and overall variable treatment costs based on water quality, and to subsequently link such model to an algorithm able to identify the most economical raw water source (i.e. reservoir/depth)

3. To develop medium-term storage volume prediction models for the two reservoirs to quantify depletion and spill risks, based on planned withdrawal amounts (consequence of intake selection model outputs)

4. To develop graphical user interfaces (GUI) and a smartphone application to facilitate the models' deployment by the WTP operators.

As most of the findings of Objectives \#1, \#2 and \#3 have been discussed elsewhere (Bertone et al., 2017a; Bertone et al., 2017b), this paper mainly focuses on Objective \#4.

\section{METHODS}

\subsection{Research location}

The Mudgeeraba WTP (Figure 1) is the second largest WTP in the Gold Coast region, South-East Queensland Australia, after the Molendinar WTP which withdraws water from the lower intake of Hinze dam. The Mudgeeraba WTP has a standard water treatment train with two conventional clarifiers and 16 mono media sand filters (Rogers et al., 2008). Raw water can be taken from the upper intake of Hinze dam (HUI, Fig. 1), through three electric pumps (74 ML/day maximum that can reach the WTP); the other withdrawal location is Little Nerang dam (LND, Fig. 1). The two offtakes towers (5 gates in LND, 9 gates in HUI) are, respectively, $3 \mathrm{~km}$ and $8 \mathrm{~km}$ away from the WTP. Hinze dam has also another intake tower, which redirects water to the Molendinar WTP.

Hinze dam is the main source of raw water for the Gold Coast region, and it has a maximum capacity of 310,700 ML, while Little Nerang dam has a maximum capacity of only 6,705 ML - which however, assuming no rain, is enough for supplying raw water to the WTP for three months (Hamilton, 2015). Given its small capacity and the fact it is rarely used for prolonged periods of time, LND often spills during wet weather events. Hinze dam on the other hand, is a much larger, stable reservoir that destratifies only in winter (Bertone et al., 2015b), creating some water quality issues (e.g. manganese, Bertone et al. (2015a)) in the epilimnion, which has otherwise typically acceptable water quality, thus allowing for regular withdrawal from this top layer. 


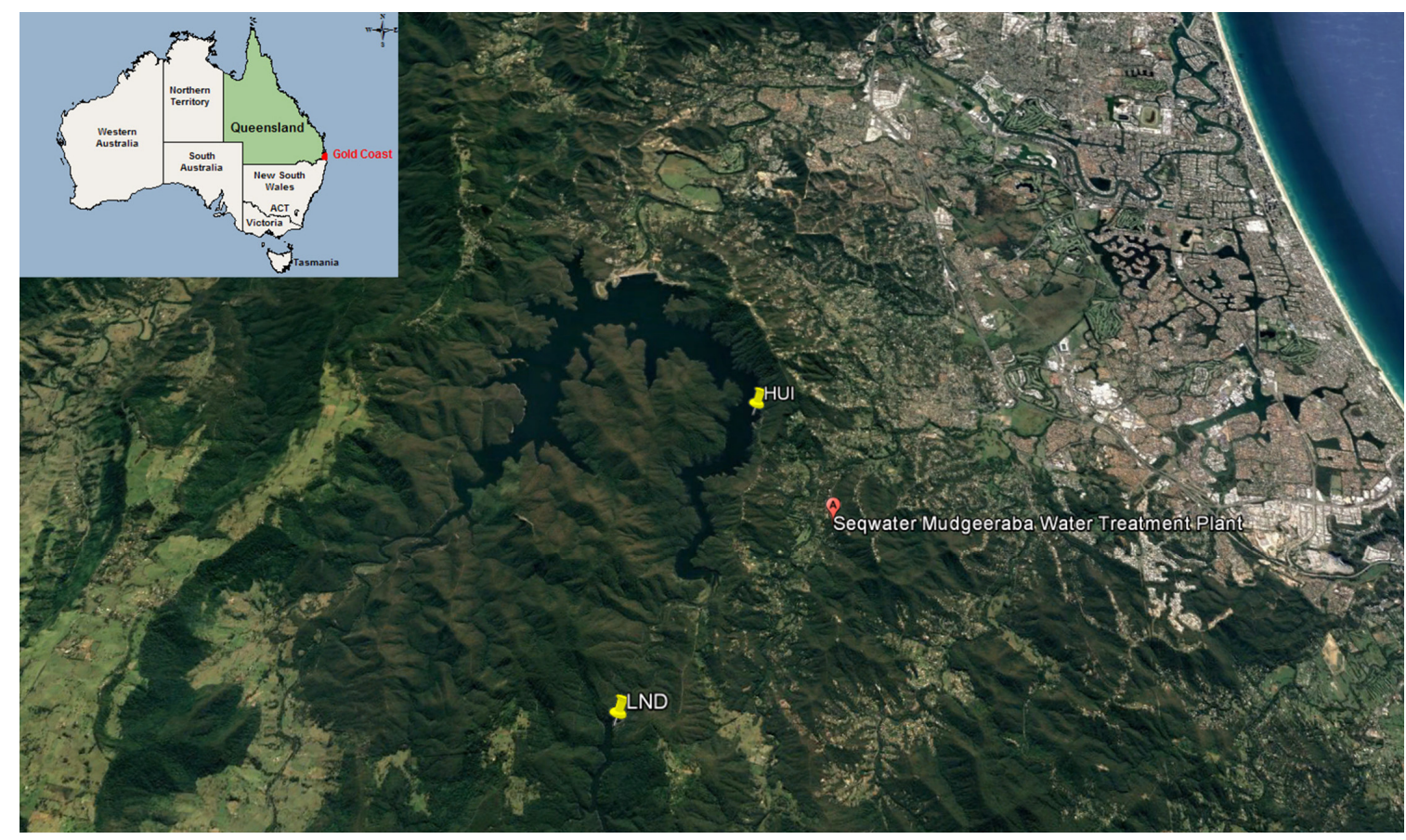

Figure 1. Location of Mudgeeraba WTP and the two intake towers in HUI and LND

\subsection{Intake optimisation model development}

Historical data were collected from a number of sources. Data included: daily chemicals dosages; raw water quality (WQ) parameters such as turbidity, $\mathrm{pH}$, dissolved oxygen, manganese, water temperature, water colour, and alkalinity; reservoir water quality (weekly lake sampling and hourly vertical profilers data); and daily flow from LND and HUI. The period were data was consistently available was 2010-14. Data were analised with self-organizing maps (Kohonen, 1998) and linear/nonlinear regression approaches. Operators were engaged to understand and justify the correlations found, and a number of models (data-driven, chemical, mathematical), sequentially predicting the required amount of each chemical based on the actual temporal dosing order (according to plant processes), as well as the variation in water quality (e.g. $\mathrm{pH}$ ) after each step, was developed. Pumping costs were also modelled based on the inflow from HUI. The full methodology is presented in Bertone et al. (2016b). A Monte Carlo approach was then applied, where 100,000 simulations were run to identify the best blend of raw water sources.

\subsection{Medium-term storage volume prediction model}

After the intake optimisation model was developed and validated, it was applied over historical data to check what the best intake selection would have been, and it was found that, due to the cheaper intake method (i.e. gravity vs. pumps), LND would have represented in several circumstances (i.e. excluding very few occasions of much worse water quality, and few long dry periods where its level would have decreased too much if the maximum withdraw rate had been applied for a prolonged period) the optimal reservoir selection. As a consequence, in order to facilitate a change of mindset whereby the operators are worried of potential depletion risk, a model was developed to predict 6 weeks ahead the likely LND volume and related spill and depletion risks. As the electricity billing system in place implies that one single daily use of the HUI pumps within a month would trigger extremely, proportionally, high fixed charges, this model allows the plant decision-maker, towards the end of a given month, to quantify the risk of LND depletion if this was to be selected as the only source of raw water.

In order to develop such model, data were collected from a number of sources, and included historical LND water level, river inflow, outflows (e.g. spills, environmental flow, WTP withdrawals). Additionally, the Bureau of Meteorology (BoM)'s Seasonal Streamflow Forecasts (SSF), providing the likelihood of certain Australian rivers to supply a below-, near- or above-median flow for the upcoming three months, were used as a key-input for prediction. A probabilistic, Monte Carlo based approach was deployed also in this circumstance. The full methodology is described in Bertone et al. (2017a). Essentially, the inflow amount used in each simulation was based on the SSF, i.e. if the SSF predicted a $30 \%$ chance of below-median 
inflow, then $30 \%$ of the model simulations would rely on a randomly generated inflow value constrained to be below median (based on historical data analysis).

\subsection{Graphical user interface and smartphone application development}

A critical component of any successful applied research project is to make sure the scientific outputs are transferred to real-life situation to create a benefit for the stakeholders. Engaging stakeholders since the model development stage (e.g. Bertone et al. (2016a)) is critical in order to agree on which outputs would be easy to understand and deploy, and therefore on which key features a decision support system (DSS) or a GUI should have. If key-stakeholders were not properly engaged throughout the project, a likely outcome would be a lack of trust in the model given his complexity although scientifically validated (Rizzoli and Young, 1997); this seems to be the case in most DSS development - related projects, with only few of them in actual use due to lack of transparency (van Delden et al., 2011).

In this case, a number of meetings were organised with Mudgeeraba WTP's operators and managers, to make sure the methodology adopted to develop the model was clear, and to obtain input and feedback on the GUI design and features; thus ensuring that the final product matched their expectation, needs, and skills (McIntosh et al., 2011). Such GUI was developed in a Matlab environment through the Matlab Compiler. Inputs entered through the GUI feed the code, which is run through a button of the GUI. A number of output charts are then displayed. Figure 2 summarises the methodological process adopted for this project in which critical stakeholders were engaged from data collection to GUI development.

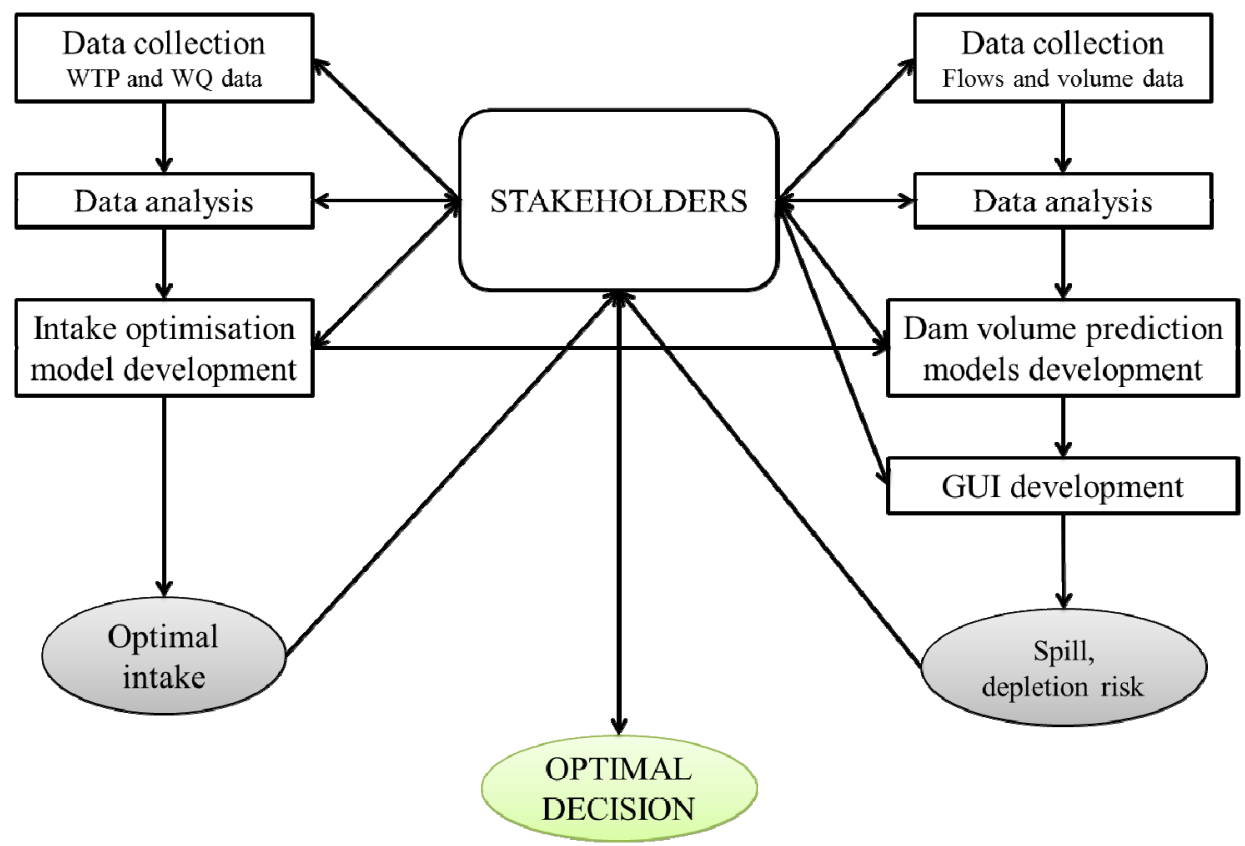

Figure 2. Conceptual diagram for models' development and deployment

Compared with a Matlab application, an application based on smartphone enjoys more popularity among people without engineering knowledge and skills. Hence, based on the developed Matlab GUI, the Research Team developed an Android Application to make it more accessible to stakeholders with different backgrounds and roles. Firstly, the core algorithm written in Matlab was converted into Java, as Android deploys Java as its developing language. The outcome is a Java class taking the same input parameters as that of the Matlab GUI, and providing the output of analysed results. Secondly, we implemented an Android interface by using AndroidStudio which is the de facto integrated development environment for Android applications. To draw the charts for the results, we used a third-party library called MPAndroidChart.

\section{RESULTS}

\subsection{Modelling results and performance}

The chemicals dosage prediction models all achieved high accuracy, with alum, polydadmac (i.e. polydiallyldimethylammonium chloride) and sodium hypochlorite predictions being the most accurate, lime and carbon dioxide predictions slightly underestimating the real values, and sodium hydroxide predictions 
yielding the lowest accuracy (Bertone et al., 2016b). However, the overall treatment cost was predicted with acceptable accuracy $\left(\mathrm{R}^{2}>0.7\right)$ and, despite still requiring operators to run jar tests for an exact estimation of the required dosage, the accuracy of the model is enough to achieve its primary goal, i.e. being able to compare and identify, among 14 options (i.e. the 9 gates' depths of HUI and the 5 gates' depths of LND), the best intake depth.

The LND storage prediction model was validated under a range of totally different historical conditions (e.g. full/dry periods; high/low withdrawal rates; etc.) with the best final model able to identify the correct most likely volume interval and the most likely spill risk (i.e. high/low/none) in over $80 \%$ of the cases (Bertone et al., 2017a). For completeness, a similar model was developed for Hinze dam too; however it is of very limited significance to the WTP operators, as its proportionally much (i.e. 46 times) higher storage capacity makes a potential depletion risk much more unlikely, or at least less influenced by the comparatively more insignificant withdrawal amounts.

\subsection{GUI and smartphone application functionalities}

Figure 3 shows the GUI main screen for both LND and Hinze dam. The inputs required are: the month of the year, the current water level, the SSF (a link is provided to the BoM webpage where such forecasts can be found), and the planned intake to the WTP (Mudgeeraba WTP only for LND, Mudgeeraba + Molendinar WTPs for Hinze dam). The big "simulation" button runs the code, generating the three output charts, namely: (1) a histogram showing the predicted distribution of the future storage level, (2) a pie chart displaying the risk of spill and (3) a pie chart displaying the probability of the storage level being above or below certain thresholds. The thresholds were defined based on operators' input and hydraulic constraints.

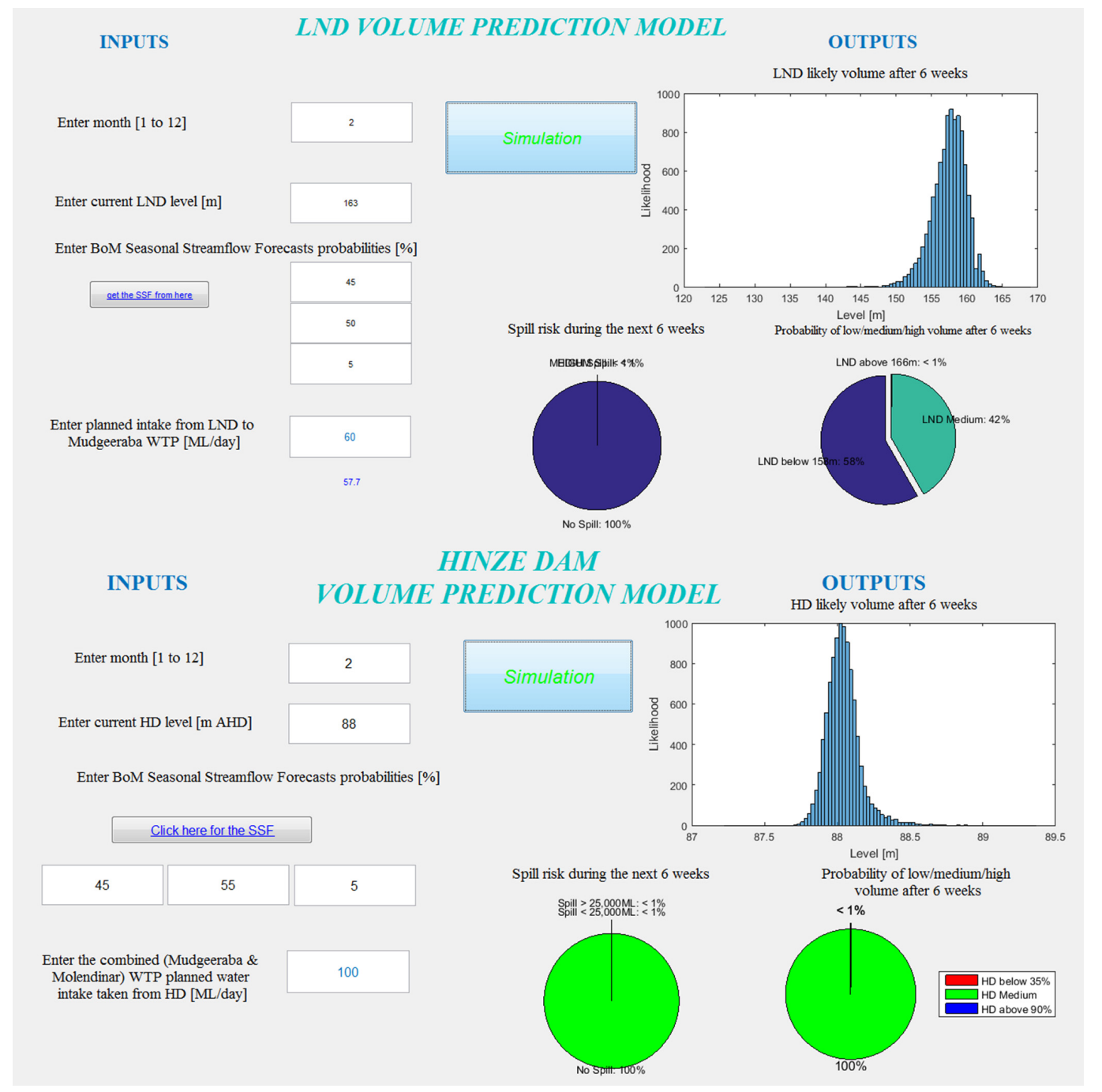

Figure 3. GUI main screen for LND (above) and Hinze dam (below) 
The relevance of this tool for LND, compared to Hinze dam, is highlighted in the figure, as the simulation results are for similar background conditions (i.e., dry conditions, medium initial storage volume, relatively high withdrawal rates). It can be noticed from the histogram how the predicted LND volume spans around an interval of $15 \mathrm{~m}$, while Hinze dam's is concentrated around $88 \mathrm{~m}$ above sea level. It can be also seen from the pie charts how there is a high chance that LND will be below critical levels (i.e. high depletion risk), while Hinze dam would not be considerably affected by one single month of dry conditions and high outflows. Such a high chance of low LND capacity is confirmed by the fact that it would not be even possible to withdraw the whole proposed daily amount of raw water (i.e. $60 \mathrm{ML}$ ) since, due to hydraulic issues (such as head loss), only 57.7 ML/day on an average (blue number in Figure 3) could be withdrawn. Based on that, the operator can run a new simulation, where a smaller intake amount is proposed, in order to reduce the depletion risk to safer levels.

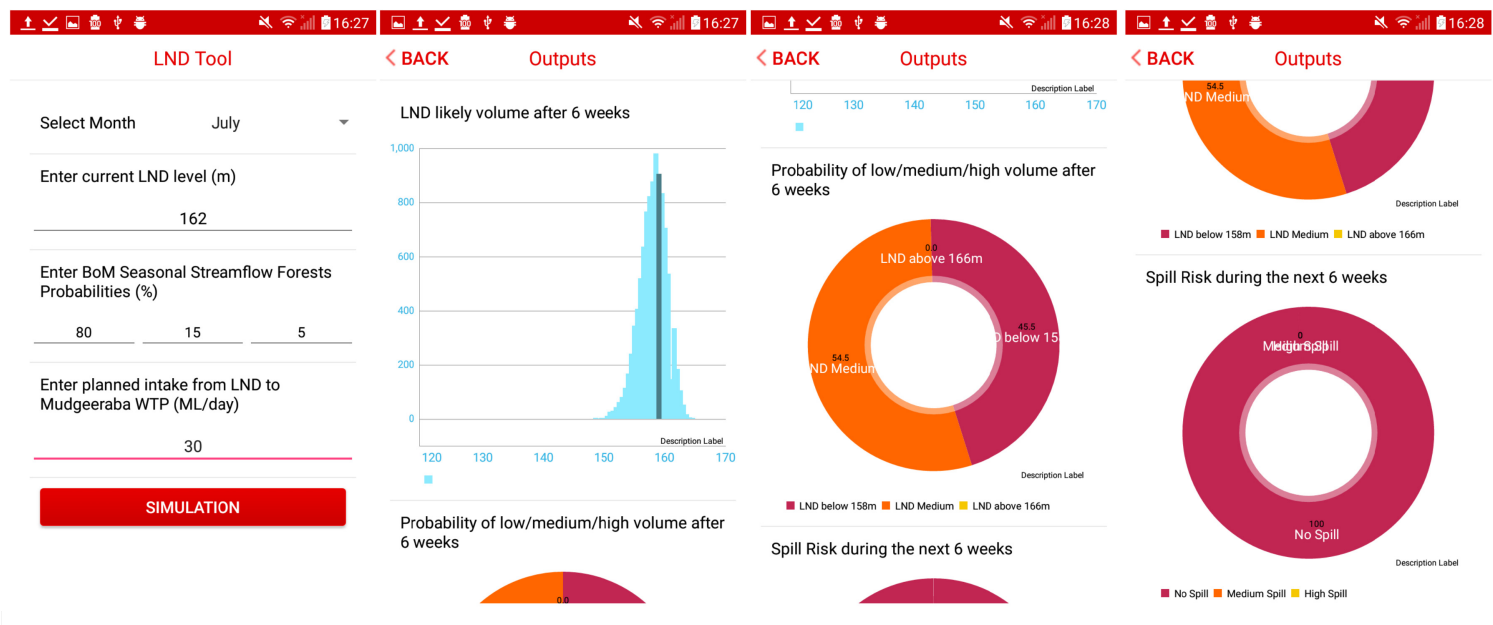

Figure 4. Screenshot for Android Application

Figure 4 shows a screenshot of the developed Android Application. It takes the same input as that of the Matlab GUI. The user could select the month from a handy dropdown menu. All the values are required to be provided in order to activate the simulation button to further analyse the data. One advanced feature of the Android Application is that it automatically grabs the BoM streamflow forecasts probabilities from the BoM website and fill the forms for the user, while the user can still override those values. In addition, as the core algorithm was converted from Matlab into Java, the application runs almost instantaneously and in general it is much faster than the computer-based Matlab GUI.

The Android Application has been delivered to the treatment plant operators during winter 2017; during the last week of each calendar month, the application is run in order to assess the depletion risk associated with a potential decision of withdrawing $100 \%$ of the required raw water from LND for the following calendar month - in order to avoid the pumping costs associated with withdrawing from HUI. If the risk is deemed acceptable, only LND is used for the next calendar month, leading to energy savings of tens of thousands of Australian dollars.

\section{CONCLUSIONS}

A number of prediction models were developed in order to optimise treatment operations in a South-East Queensland dual source water treatment plant. An intake optimisation model was firstly developed, which could calculate the treatment (i.e. chemicals, electricity) costs based on selected reservoir and water quality. Secondly, two medium-term water level forecasting models were developed for the two source reservoirs, in order to enable the operators to safely select the optimal intake location without incurring in unacceptable spill or depletion risks for these reservoirs.

The models were developed using different methodologies. The overall intake optimisation model was developed by combining a number of data-driven, chemical and mathematical models for cost estimation, with a final Monte Carlo algorithm deployed to identify the optimal intake location. The 6-week ahead water level forecasting models were based on a probabilistic Monte Carlo based approach due to the large inputs uncertainty, including BoM weather forecasts. 
Much effort in this particular study has been dedicated to the models' deployment aspect. Firstly, a GUI was developed, which allows stakeholders to understand and run the model through a user-friendly environment. However, a smartphone application was also developed to further enhance the deployment of the model, especially by stakeholders and operators spending most of their working day on field. Given that a relatively large proportion of water utilities' staff members work in multiple locations, and thus have a much easier access to smartphones compared to computers, there is potential to develop other smartphone applications to translate previously completed scientific research work into user-friendly, easily accessible, useful information.

It was estimated that an optimised selection of the raw water intake for the Mudgeeraba WTP, which often implies an increased use of the smaller LND, would lead to remarkable monetary savings for Seqwater. Thus future work will aim at ensuring the model is regularly used, and such savings achieved.

\section{ACKNOWLEDGMENTS}

The Authors would like to acknowledge Seqwater and the Cities Research Institute for financial and technical support for this project, and Mr. Maverick-Benjamin Jeske for helping with data collection and water level model development for Hinze dam. The Authors are also grateful to Seqwater staff members (e.g. treatment plant operators and managers) for the time they dedicated to provide suggestions and feedback during the course of the project.

\section{REFERENCES}

Abdullahi, M. (2013). Development of a model for the estimation of water treatment cost: a data mining approach. Int. J. Sci. Eng. Res 4, 610-615.

Bertone, E., O' Halloran, K., Stewart, R.A., de Oliveira, G.F. (2017a). Medium-term storage volume prediction for optimum reservoir management: A hybrid data-driven approach. Journal of Cleaner Production 154, 353-365.

Bertone, E., O'Halloran, K., Bartkow, M., Mann, K. (2017b). Autonomous intake selection optimisation model for a dual source drinking water treatment plant. Water Science and Technology: Water Supply, ws2017111.

Bertone, E., Sahin, O., Richards, R., Roiko, A. (2016a). Extreme events, water quality and health: A participatory Bayesian risk assessment tool for managers of reservoirs. Journal of Cleaner Production $135,657-667$.

Bertone, E., Stewart, R.A., Zhang, H., Bartkow, M., Hacker, C. (2015a). An autonomous decision support system for manganese forecasting in subtropical water reservoirs. Environmental Modelling \& Software 73, 133-147.

Bertone, E., Stewart, R.A., Zhang, H., O'Halloran, K. (2016b). Hybrid water treatment cost prediction model for raw water intake optimization. Environmental Modelling \& Software 75, 230-242.

Bertone, E., Stewart, R.A., Zhang, H., O’Halloran, K. (2015b). Analysis of the mixing processes in the subtropical Advancetown Lake, Australia. Journal of Hydrology 522, 67-79.

Hamilton, G. (2015). Validation of LND Gravity Main Capacity. Technical Memorandum \#1.: Report prepared for Seqwater by GH Consultant Engineers, August 2015.

Kawamura, S. (2000). Integrated design and operation of water treatment facilities: John Wiley \& Sons.

Kohonen, T. (1998). The self-organizing map. Neurocomputing 21, 1-6.

McIntosh, B.S., Ascough, J.C., Twery, M., Chew, J., Elmahdi, A., Haase, D., Harou, J.J., Hepting, D., Cuddy, S., Jakeman, A.J., Chen, S., Kassahun, A., Lautenbach, S., Matthews, K., Merritt, W., Quinn, N.W.T., Rodriguez-Roda, I., Sieber, S., Stavenga, M., Sulis, A., Ticehurst, J., Volk, M., Wrobel, M., van Delden, H., El-Sawah, S., Rizzoli, A., Voinov, A. (2011). Environmental decision support systems (EDSS) development - Challenges and best practices. Environmental Modelling \& Software 26, 13891402.

Rizzoli, A.E., Young, W.J. (1997). Delivering environmental decision support systems: software tools and techniques. Environmental Modelling \& Software 12, 237-249.

Rogers, P., Lockyer, J., Stevenson, S. (2008). Mudgeeraba WPP filtration evolution. 33rd Annual Qld Water Industry Operations Workshop, Indoor Sports Centre, Carrara, Gold Coast City, Australia.

van Delden, H., Seppelt, R., White, R., Jakeman, A.J. (2011). A methodology for the design and development of integrated models for policy support. Environmental Modelling \& Software 26, 266279. 